\title{
Cartilage Imaging in Osteoarthritis
}

\author{
Hamza Alizai, MD ${ }^{1,2} \quad$ William Walter, MD ${ }^{1} \quad$ Iman Khodarahmi, MD ${ }^{1} \quad$ Christopher J. Burke, MBChB \\ 1 Department of Radiology, NYU Langone Health, New York, New York \\ 2 Texas Scottish Rite Hospital for Children, Dallas, Texas \\ Address for correspondence Hamza Alizai, MD, Department of \\ Radiology, NYU Langone Health, 660 1st Avenue, New York, NY, 10016 \\ (e-mail: hamzaalizai@gmail.com).
}

Semin Musculoskelet Radiol 2019;23:569-578.

\begin{abstract}
Osteoarthritis $(\mathrm{OA})$ is the most common joint disease in the United States. The prevalence of $\mathrm{OA}$ is rising due to an aging population and increasing rates of obesity. Magnetic resonance imaging (MRI) allows an incomparable noninvasive assessment of all joint structures. Irreversible and progressive degradation of the articular cartilage remains the hallmark feature of $O A$. To date, attempts at developing disease-modifying drugs or biomechanical interventions for treating OA have proven unsuccessful. MRIbased cartilage imaging techniques have continued to advance, however, and will likely play a central role in the development of these joint preservation methods of the

Keywords

- cartilage

- MRI

- quantitative

- semiquantitative future. In this narrative review, we describe clinical MR image acquisition and assessment of cartilage. We discuss the semiquantitative cartilage scoring methods used in research. Lastly, we review the quantitative MRI techniques that allow assessment of changes in the biochemical composition of cartilage, even before the morphological changes are evident.
\end{abstract}

Osteoarthritis $(\mathrm{OA})$ is the most common joint disease in the United States, and its prevalence is rising due to an aging population and increasing rates of obesity. ${ }^{1,2}$ Irreversible and progressive degradation of the articular cartilage remains the fundamental feature of OA pathophysiology. Conventional radiography is considered the reference standard for imaging of OA; however, joint space narrowing (JSN) on radiography provides an indirect measure of cartilage loss and is not sensitive to progression of the disease. ${ }^{3-5}$ Radiography-based JSN is nonetheless commonly used as the imaging outcome measure to establish the effectiveness of disease-modifying osteoarthritis drugs (DMOADs). ${ }^{6}$ The use of radiographic JSN as an outcome measure may partly explain why attempts at developing DMOADs and behavioral therapy for OA have proven unsuccessful, despite promising preclinical research. ${ }^{7}$

Regulatory agencies including the U.S. Food and Drug Administration (FDA) are increasingly recommending imaging beyond radiography to assess early onset of abnormalities in OA. ${ }^{8}$ The multiplanar, multiparametric capabilities of magnetic resonance imaging (MRI) and its excellent soft tissue contrast allow unparalleled evaluation of all joint structures including cartilage. Hence MRI-based outcome measures are ideal for

(D) Hamza Alizai's ORCID is https://orcid.org/0000-0001-6498-1774.

Issue Theme Imaging Around the Ends of Bones; Guest Editor, Christian Glaser, MD assessment of cartilage degradation in OA. In addition to the routine clinical MRI, advanced research techniques have been developed to assess the biochemical composition of cartilage in the earliest stages of OA. These include relaxometry measurements (T2, T2*, and T1 $\rho$ mapping), sodium imaging, delayed gadolinium-enhanced MRI of cartilage (dGEMRIC), glycosaminoglycan specific chemical exchange saturation transfer (gagCEST), and diffusion tensor imaging (DTI). These compositional MRI techniques serve as quantitative, reproducible, and objective end points for OA research that will likely be introduced to clinical radiology practice in the near future.

In this narrative review, we describe the standard clinical imaging of cartilage in OA. We discuss semiquantitative scoring systems for the assessment of cartilage that serve as important outcome measures in research. Lastly, we highlight advanced compositional MRI techniques that allow the detection of early articular cartilage degradation.

\section{Cartilage Microarchitecture}

Understanding the imaging of chondral degeneration in $\mathrm{OA}$ requires an understanding of the basic ultrastructure of the articular cartilage. It is composed of primarily fluid (70-80\%) and extracellular matrix (ECM), both of which are essential

Copyright (c) 2019 by Thieme Medical Publishers, Inc., 333 Seventh Avenue, New York, NY 10001, USA. 
for its normal function. ${ }^{9}$ The ECM is a network of collagen fibrils and proteoglycan molecules, with the proteoglycan consisting of negatively charged glycosaminoglycan (GAGs) attached to the protein core. ${ }^{9}$ The negative charge attracts and holds water within articular cartilage while cations such as sodium $\left(\mathrm{Na}^{+}\right)$counter the negative charge of GAGs. The redistribution of water within the ECM provides the known biomechanical properties of cartilage, that is, its ability to deal with tensile and compressive loads. ${ }^{10}$ In OA, proteoglycan loss and disorganization and/or loss of the collagen fiber network lead to impaired ability of the articular cartilage to deal with these loads and results in progressive, irreversible breakdown. $^{11}$

\section{Clinical MRI of Cartilage}

An in-plane resolution of $0.3 \mathrm{~mm}$ resolves the earliest stage of morphological cartilage degeneration, that is, fraying of the articular surface. ${ }^{12}$ Optimal evaluation of cartilage morphology on standard clinical MRI, therefore, requires high signal-tonoise ratio (SNR) and a high spatial resolution, both of which are advantages of higher field strength magnets $(\geq 1.5 \mathrm{~T}$, with dedicated extremity coils). A recent systemic review and meta-analysis comparing 1.5-T and 3-T MRI for detection of morphological cartilage lesions found both field strength magnets to offer high diagnostic accuracy; however, the 3-T MRI had greater accuracy than the $1.5 \mathrm{~T}^{13}$ In 2017 , the FDA approved the first 7-T MRI system for clinical diagnostic imaging of the extremities. A comparison of routine clinical knee MRI performed at $3 \mathrm{~T}$ and $7 \mathrm{~T}$ found diagnostic confidence of radiologists for cartilage defects to be higher with $7 \mathrm{~T}^{14}$

In addition to adequate SNR and spatial resolution, detection of cartilage pathology requires optimal cartilage-synovial fluid contrast. The International Cartilage Repair Society (ICRS) protocol for imaging of cartilage includes two-dimensional (2D) fast spin-echo (FSE) or turbo spin-echo (TSE) pulse sequences to obtain fat-suppressed proton-density-weighted, T2-weighted, or intermediate-weighted images. ${ }^{15}$ These sequences provide excellent tissue contrast allowing detection of cartilage lesions with high accuracy; however, they require acquisition in multiple planes. - Fig. 1 shows fat-suppressed proton-density images of the patellofemoral compartment cartilage in a healthy volunteer (-Fig. 1a) and in a patient with advanced osteoarthritis (-Fig. 1b). Isotropic sequences (3D FSE or TSE) obviate the need for multiplanar acquisition, greatly reducing acquisition time. ${ }^{16}$ Isotropic imaging suffers from blurring and lower tissue contrast compared with 2D sequences; however, the diagnostic accuracy of isotropic FSE for cartilage morphology was shown to be similar to 2D FSE at $3 \mathrm{~T} .{ }^{17}$

Cartilage-sensitive techniques based on gradient spin-echo (GRE) such as 3D spoiled gradient recalled echo produce images with cartilage signal more intense than the surrounding tissues, which renders them insensitive to subtle cartilage lesions and of limited utility in clinical imaging. ${ }^{18}$ These techniques were used successfully for quantitative assessment of cartilage thickness and volume in research studies. ${ }^{19-21}$

\section{Semiquantitative Assessment of Cartilage}

Semiquantitative scoring systems for cartilage including the Outerbridge $(1961)^{22}$ and Noyes and Stabler $(1989)^{23}$ classifications were originally developed for grading the appearance and quantity of cartilage via direct evaluation during surgery. The ICRS classification, a 9-point scale, succeeded these initial scoring systems ${ }^{24}$ and provided a more comprehensive and detailed assessment of articular cartilage pathology. All of these classifications have been adapted for assessment of joint cartilage on MRI, ${ }^{25-27}$ primarily for research.

As the use of MRI for OA was researched and increased, dedicated MRI-based semiquantitative classifications for whole-organ assessment of joints, most commonly the knee joint, were developed and validated. In the next section, we
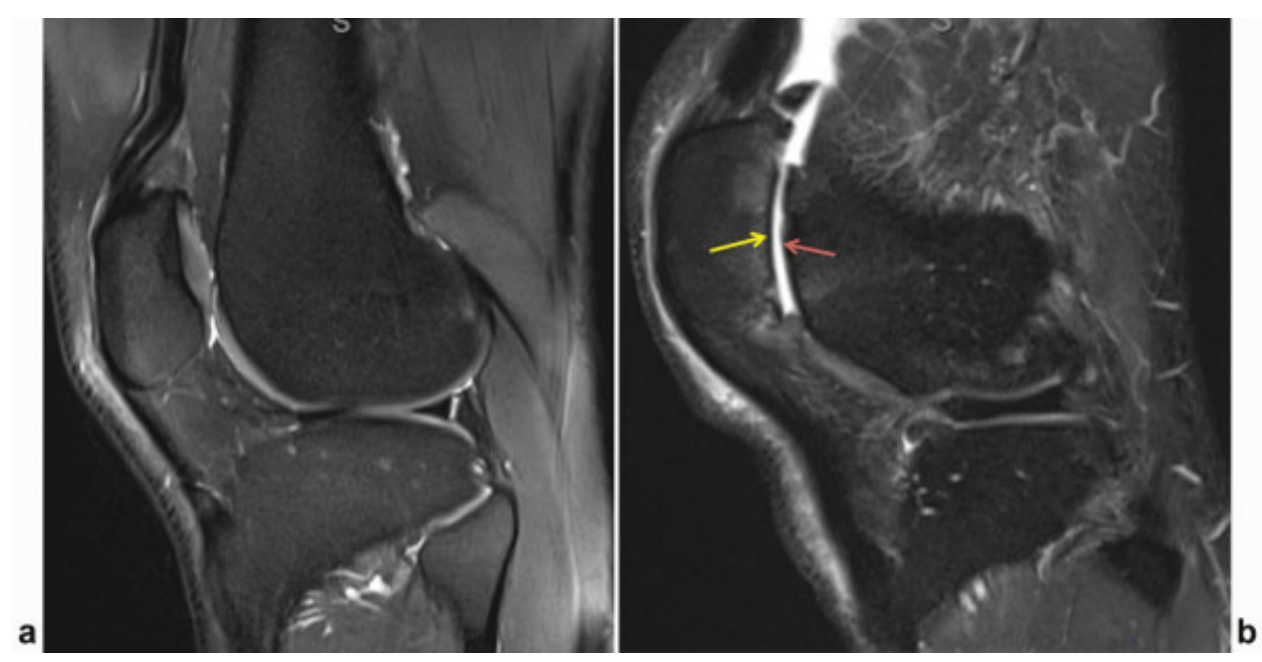

Fig. 1 Sagittal fat-suppressed proton-density images of the patellofemoral compartment cartilage in (a) a healthy volunteer and (b) a patient with advanced osteoarthritis (OA). The healthy volunteer has normal patellar and femoral trochlear cartilage thickness and signal density. The patient with OA demonstrates full-thickness cartilage loss overlying the patella (yellow arrow) and overlying the femoral trochlea (red arrow), with associated subchondral bone marrow edema. 
briefly describe a select few of these systems that are most likely to be encountered in the published literature.

\section{Whole-Organ Magnetic Resonance Imaging Score}

The Osteoarthritis Initiative (OAI) and the Multicenter Osteoarthritis Study (MOST) are two of the largest multicenter and longitudinal studies of OA that included MRI acquisition in addition to expansive clinical data for the study of OA. ${ }^{28-33}$ The Whole-Organ Magnetic Resonance Imaging Score (WORMS) is the most commonly used scoring system in knee $O A$ research and was used as an outcome measure in the OAI and MOST. The WORMS assesses 14 features in the knee joint that include articular cartilage integrity, subchondral bone marrow abnormality, cruciate ligament, and meniscal integrity among other features. ${ }^{34}$ Cartilage is graded on an 8point scale in 14 subregions subdivided by anatomical landmarks. Cartilage grades in the different subregions are frequently summed to provide composite or global cartilage scores (-Fig. 2).

\section{Boston-Leeds Osteoarthritis Knee Score}

Concerns about the responsiveness of WORMS and the validity of summation of subregional WORMS cartilage measurements led to the development of the Boston-Leeds Osteoarthritis Knee Score (BLOKS). ${ }^{35}$ The BLOKS evaluates cartilage in nine subregions of the knee. BLOKS I cartilage score, the more commonly used of the two-part cartilage scoring component of BLOKS, assesses cartilage on a 4-point scale. It assigns separate scores for (1) the areal extent of any cartilage loss in each subregion, and (2) the percentage of subregion surface area that has a full-thickness loss (-Fig. 2).

\section{MRI Osteoarthritis Knee Score}

Both WORMS and BLOKS have limitations, highlighted in a two-part study comparing these methods. ${ }^{36,37}$ As a result, the MRI Osteoarthritis Knee Score (MOAKS) was derived from both the BLOKS and WORMS to improve whole-organ assessment of the knee. ${ }^{38}$ MOAKS grades cartilage in the same 14 subregions of the knee as are graded in WORMS, but it uses the grading scale used in the BLOKS "cartilage I" score (-Fig. 1).

\section{Knee Osteoarthritis Scoring System}

The Knee Osteoarthritis Scoring System ${ }^{39}$ is another wholeorgan grading system focused on the knee that grades cartilage in nine subregions. It assigns separate 4-point scores for the depth of the cartilage and osseous components of an osteochondral defect. It also assigns a separate 4-point score for the surface extent of an osteochondral defect estimated by its maximal diameter. A focal cartilaginous defect is well defined with an acute angle between the defect and surrounding cartilage. A diffuse defect has an obtuse angle between the normal and thinned cartilage.

\section{Use of Semiquantitative Assessment Methods in Research}

These classification schemes have been used extensively as outcome measures in research including in large multicenter trials such as the OAI and MOST. The following are select

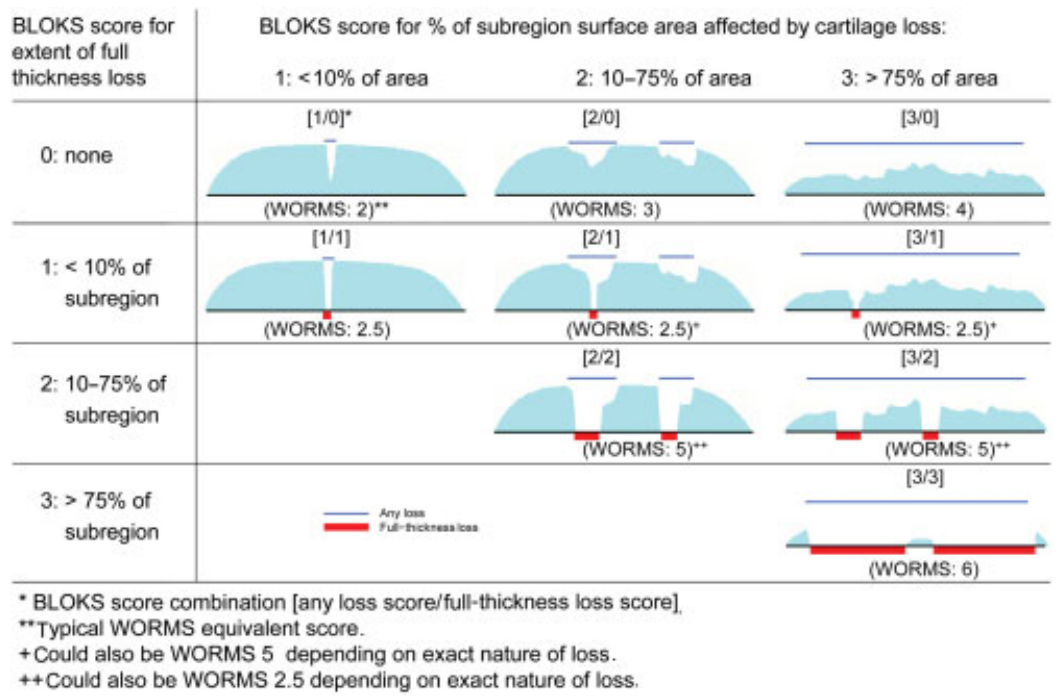

Fig. 2 Whole-organ magnetic resonance imaging score (WORMS) descriptions: $0=$ normal thickness and signal; $1=$ normal thickness but increased signal on T2-weighted images (not used in this study); 2.0 = partial-thickness focal defect $<1 \mathrm{~cm}$ in greatest width; $2.5=$ full-thickness focal defect $<1 \mathrm{~cm}$ in greatest width; $3=$ multiple areas of partial-thickness defects $<75 \%$ of region or a single partial-thickness defect wider than $1 \mathrm{~cm}$ but $<75 \%$ of the region; $4=$ diffuse ( $>75 \%$ of the region) partial-thickness loss; $5=$ multiple areas of full-thickness loss $<75 \%$ of the region or a single full-thickness lesion wider than $1 \mathrm{~cm}$ but $<75 \%$ of the region; 6 = diffuse ( $>75 \%$ of the region) full-thickness loss. Boston-Leeds Osteoarthritis Knee Score (BLOKS) descriptions: Size of any cartilage loss (including partial- and full-thickness loss) as a percentage of surface area as related to the size of each individual region: $0:$ none; $1:<10 \%$ of region of cartilage surface area; 2: 10 to $75 \%$ of region of cartilage surface area; 3: $>75 \%$ of region of cartilage surface area; and percentage full-thickness cartilage loss of the region: 0 : none; $1:<10 \%$ of region of cartilage surface area; $2: 10$ to $75 \%$ of region of cartilage surface area; $3:>75 \%$ of region of cartilage surface area. Reproduced with permission from Lynch et al. ${ }^{36}$ 
examples of studies of OA risk factors using semiquantitative assessment.

\section{Osteoarthritis and Physical Activity}

The impact of physical activity on OA remains a controversial topic. Although some studies found exercise to be beneficial, ${ }^{40,41}$ studies of patients from the OAI contradicted these findings. In particular, these studies of OAI patients reported that individuals who have risk factors for OA may suffer cartilage degeneration with high-intensity physical activity. ${ }^{29,42,43}$ Even in asymptomatic individuals enrolled in the OAI, cartilage lesions were more common and more severe in the highly active subjects compared with the less active subjects. ${ }^{29,44}$ In patients who have knee abnormalities at baseline, walking $>10,000$ steps per day was associated with higher cartilage defect scores. ${ }^{45}$ Physical activity involving frequent knee bending was also implicated in a higher prevalence of knee cartilage lesions and increased the progression of these lesions, particularly in the patellofemoral compartment. ${ }^{33}$

\section{Obesity}

In obese adults, knee cartilage defects are associated with physical disability. ${ }^{46}$ In the OAI cohort, obesity was associated with a higher prevalence and severity of knee cartilage lesions as well as with increased cartilage lesion progression over 3 years. ${ }^{32,47}$ High body mass index was also associated with rapid tibiofemoral cartilage loss in patients enrolled in MOST who had or were at risk for OA. ${ }^{48}$ Weight loss may help prevent development/progression of lesions and improve quality of life. ${ }^{49}$

\section{Injuries}

In the OAI cohort, individuals with anterior cruciate ligament (ACL) abnormalities had a greater prevalence of cartilage lesions that were also more severe compared with individuals with a normal ACL. ${ }^{30}$ Meniscal tears were also found to be associated with poor tibiofemoral cartilage scores, even in patients without OA. ${ }^{50}$ Meniscal root tears are particularly implicated. $^{51,52}$ The presence of meniscal extrusion is also associated with the prevalence and severity of cartilage damage. ${ }^{53,54}$

\section{Alignment}

Knee malalignment in either the valgus or varus direction affects the distribution of the load across the joint. ${ }^{55} \mathrm{~A}$ large study examined 5,053 knees from the MOST and 5,953 knees from the OAI cohort using either WORMS or BLOKS. ${ }^{56}$ This study found valgus malalignment, particularly $>3$ degrees, to be associated with an increased risk of cartilage defect progression in the lateral tibiofemoral compartment. In the MOST cohort, varus malalignment has, in contrast, been associated with incident cartilage damage in the medial compartment. ${ }^{57}$

\section{Compositional MRI Techniques for Assessment of Cartilage}

Primarily used in research, these techniques allow detection of the earliest changes of cartilage degeneration in the ECM, well before the morphological cartilage defects are apparent. Thus, compositional imaging sequences have the potential to serve as quantitative imaging biomarkers of $\mathrm{OA}$.

Higher field strength magnets ( $3 \mathrm{~T}$ and $7 \mathrm{~T}$ ) are particularly useful for compositional imaging, even more so than for clinical imaging of cartilage. These MR units afford higher SNR with resultant greater spatial resolution and shorter MRI acquisition times. ${ }^{58}$ Additionally, many biologically relevant nuclei in addition to ${ }^{1} \mathrm{H}$, such as sodium and phosphorous, occur in relatively low concentrations and warrant high field strength magnets to allow signal acquisition. Among numerous challenges, impediments to routine use of higher field strength MRI include increasing inhomogeneity, changes in relaxation times, ${ }^{59}$ increased sensitivity to susceptibility effects (decreased $\mathrm{T}_{2}{ }^{*}$ ), ${ }^{60}$ and increase in chemical shift artifact in the frequency-encode direction. ${ }^{61}$

\section{$T_{\mathbf{2}}$ Mapping and $\mathbf{T}_{\mathbf{2}}{ }^{*}$ Mapping}

$\mathrm{T}_{2}$ mapping was obtained as part of the knee MRI acquisition protocol in the OAI and was the most widely studied of all compositional imaging techniques. ${ }^{62} \mathrm{~T}_{2}$ measurements reflect dephasing in the transverse plane after application of a radiofrequency $(\mathrm{RF})$ pulse. These measurements were found to be associated with cartilage water content and reflect an indirect measure of the ECM collagen content. ${ }^{63}$ Laminar analysis of cartilage found these measurements to be higher in the superficial layers of cartilage than in the deep layers. ${ }^{64}$ Higher $\mathrm{T}_{2}$ values were shown to predict the development of cartilage lesions. ${ }^{65}$ At our institutions, a few select surgeons request $T_{2}$ mapping as part of the preoperative MRI to identify problem areas in the cartilage before performing arthroscopy (-Fig. 3). $\mathrm{T}_{2}$ mapping can discriminate between repaired knee cartilage and adjacent healthy cartilage, ${ }^{66-68}$ and it may be particularly helpful in assessing the maturation of reparative cartilage. ${ }^{69}$

$\mathrm{T}_{2}{ }^{*}$ mapping measures transverse-plane dephasing using multiecho GRE techniques. These sequences have a shorter acquisition time but are also more vulnerable to local field inhomogeneity. ${ }^{70,71}$ Both $\mathrm{T}_{2}$ and $\mathrm{T}_{2}{ }^{*}$ are affected by the magic angle effect; that is, the values increase as the angle between collagen fibers and $\mathrm{B}_{0}$ approaches 55 degrees.

\section{T1p Mapping}

$\mathrm{T}_{1} \rho$ imaging is more challenging to acquire than $\mathrm{T}_{2}$ mapping and therefore only performed at a few select academic institutions. The imaging is difficult to acquire due to $B_{0}$ and $B_{1}$ inhomogeneity, specialized RF pulse sequence requirements, and long acquisition times that may result in high specific absorption rates (SARs). The SNR gain at $7 \mathrm{~T}$ has been used, however, to show the feasibility of acquiring high-resolution $\mathrm{T} 1 \rho$ images $\left(0.2 \mathrm{~mm}^{2}\right.$ in-plane resolution) in reasonable acquisition times 


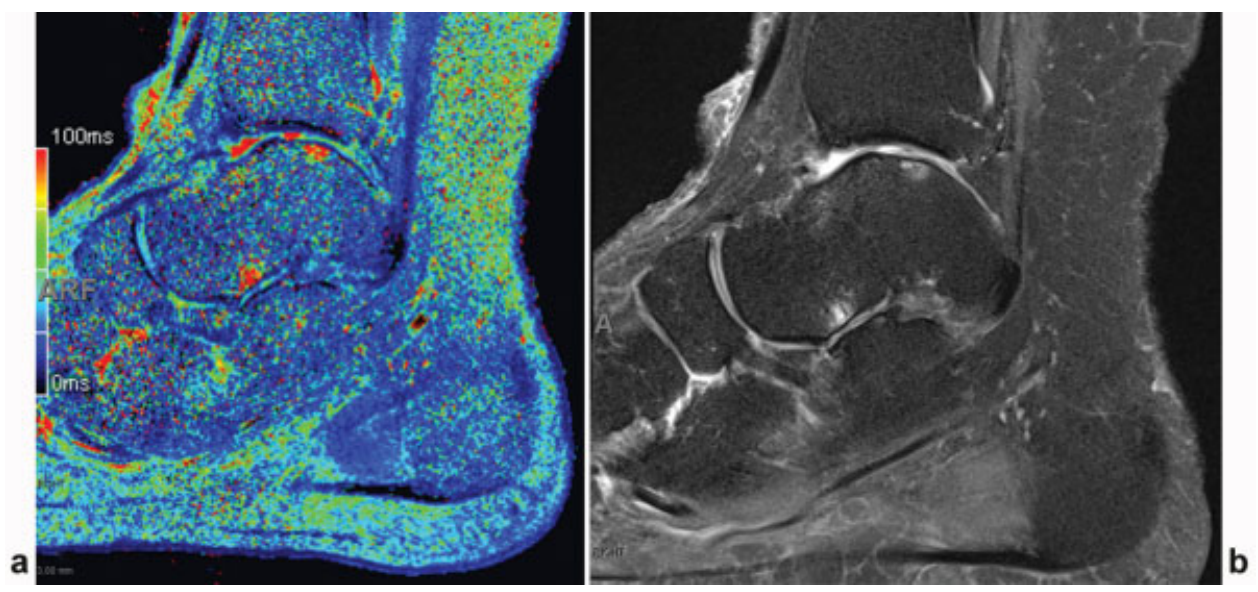

Fig. 3 T2 mapping was performed as part of the clinical preoperative MRI protocol per the request of the referring orthopaedic surgeon. (a) Sagittal T2 color map is shown with the scale provided on the left; red represents highest T2 measurements; dark blue reflects the lowest T2 values. Focal high T2 values are seen within the central tibiotalar cartilage (yellow arrow) and with the subchondral talar dome (red arrow). (b) Sagittal fat-suppressed proton-density images in the same patient demonstrate that these findings correspond to full-thickness tibiotalar cartilage loss and subchondral bone marrow edema, respectively.

( $<30$ minutes) and within SAR constraints. ${ }^{72} \mathrm{~T} 1 \rho$ assesses the spin-lattice $\left(\mathrm{T}_{1}\right)$ relaxation in the rotating frame ${ }^{73}$ and is thought to reflect the proteoglycan content of the ECM. ${ }^{74} \mathrm{~T} 1 \rho$ values are higher in patients with OA compared with healthy subjects. $^{75}$ T1 $\rho$ was also shown to predict morphological chondral wear. ${ }^{76}$

\section{Ultrashort Echo Time and Zero Echo Time Imaging}

Like cortical bone, tendons, and menisci, the deep calcified part of cartilage contains a high fraction of components with "ultrashort" transverse relaxation times. This essentially equates to a post-RF pulse signal decay rate that is too rapid to allow signal acquisition. ${ }^{77}$ Ultrashort echo time (UTE) and zero echo time use specialized acquisition and reconstruction techniques to capture these ultrashort components before signal decay. Although the application of techniques for imaging of cartilage is not common, UTE was shown to delineate the calcified deepest cartilage layer ${ }^{78}$ and used to evaluate the integrity of this layer in osteochondral allografts. ${ }^{79}$ UTE also enables $\mathrm{T}_{2}$ and $\mathrm{T}_{2}{ }^{*}$ mapping of tissues with a high fraction of ultrashort components. ${ }^{80}$

\section{Delayed Gadolinium-Enhanced MRI of Cartilage}

The dGEMRIC MRI is performed after intravenous injection of a gadolinium-based contrast with subsequent joint exercise and substantial time delay to allow diffusion of the contrast into the joint. Gadopentetate dimeglumine (Gd$\mathrm{DTPA}^{2-}$ ), the MRI contrast, is an anion and repelled by the negatively charged GAGs, allowing this technique to map GAG content within the cartilage. Damaged cartilage with low GAG content will accumulate more Gd-DTPA ${ }^{2-}$ and therefore have a shorter $T_{1}$ relaxation time. This technique was used in research to study a variety of topics including cartilage repair tissue, ${ }^{81,82}$ effects of tibial osteotomy on cartilage, ${ }^{83}$ inflammatory arthritis, ${ }^{84}$ and the effects of chronic joint unloading. ${ }^{85}$ The need for intravenous contrast is the main drawback of dGEMRIC; however, it does allow an indirect MR arthrogram to be obtained during the delay between injection and acquisition of dGEMRIC $\mathrm{T}_{1}$ imaging. This may particularly be helpful in the morphological evaluation of the acetabular or glenoid labrum.

\section{Sodium $\left({ }^{23} \mathrm{Na}\right)$ Imaging}

In contrast to Gd-DTPA ${ }^{2-}$, sodium $\left({ }^{23} \mathrm{Na}^{+}\right)$is a naturally occurring cation (albeit in very low concentrations) that is attracted to and counteracts the negatively charged GAGs in the cartilage ECM. The distribution of ${ }^{23} \mathrm{Na}^{+}$can hence also be used to map the cartilage GAG content, with cartilage degeneration resulting in a lower concentration of ${ }^{23} \mathrm{Na}^{+}$ions. ${ }^{86}$ Unsurprisingly, ${ }^{23} \mathrm{Na}$ imaging correlates well with dGEMRIC. ${ }^{87}$ The low concentrations of ${ }^{23} \mathrm{Na}^{+}$in cartilage, however, make it difficult to elicit signal during MRI, resulting in noisy images and long acquisition times. The SNR gain at 7-T MRI is particularly useful for ${ }^{23} \mathrm{Na}$ imaging. ${ }^{88}$ Because the Larmor frequency of ${ }^{23} \mathrm{Na}^{+}$differs from ${ }^{1} \mathrm{H}$, specialized transmit-receive coils are also required to perform sodium imaging. ${ }^{89}$ As with $T_{2}$ mapping, sodium imaging can discriminate between cartilage repair tissue and healthy cartilage, with lower sodium signal intensity in repair tissue compared with healthy cartilage reflecting a diminished GAG content ${ }^{90,91}$ (- Fig. 4).

\section{Diffusion Tensor Imaging}

The cartilage ultrastructure consists of a highly organized network of collagen that results in anisotropic diffusion of water. In cartilage, DTI can assess both proteoglycan content through mean apparent diffusion coefficient (ADC) and collagen microarchitecture through fractional anisotropy (FA). Both mean ADC and FA values were found to be able 


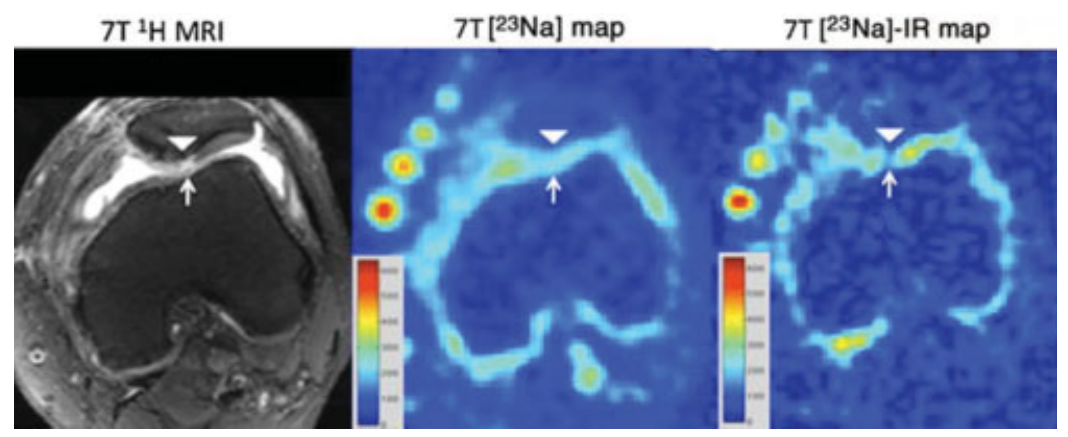

Fig. 4 Axial T2-weighted 7-T MR image (left panel) of the left knee, demonstrating a displaced osteochondral fragment reattachment (arrowhead) at the medial facet of the patella. Synovial fluid is seen within a full-thickness fissure (arrow). On the conventional ${ }^{23} \mathrm{Na}$ map (middle panel), hyperintense signal is seen from synovial fluid within the fissure (arrow). On the ${ }^{23} \mathrm{Na}-\mathrm{IR}$ map (right panel), there is suppression of signal from synovial fluid within the fissure. The calibration phantoms containing $300 \mathrm{mM}, 250 \mathrm{mM}, 200 \mathrm{mM}$, and $150 \mathrm{mM}$ are seen at the anterior aspect of the knee. The sodium images represent concentration maps with colored bars indicating range of $\left[\mathrm{Na}^{+}\right]$in $\mathrm{mM}(\mathrm{red}=600 \mathrm{mM}$; blue $=0 \mathrm{mM}$ ). Reproduced with permission from Chang et al. ${ }^{91}$
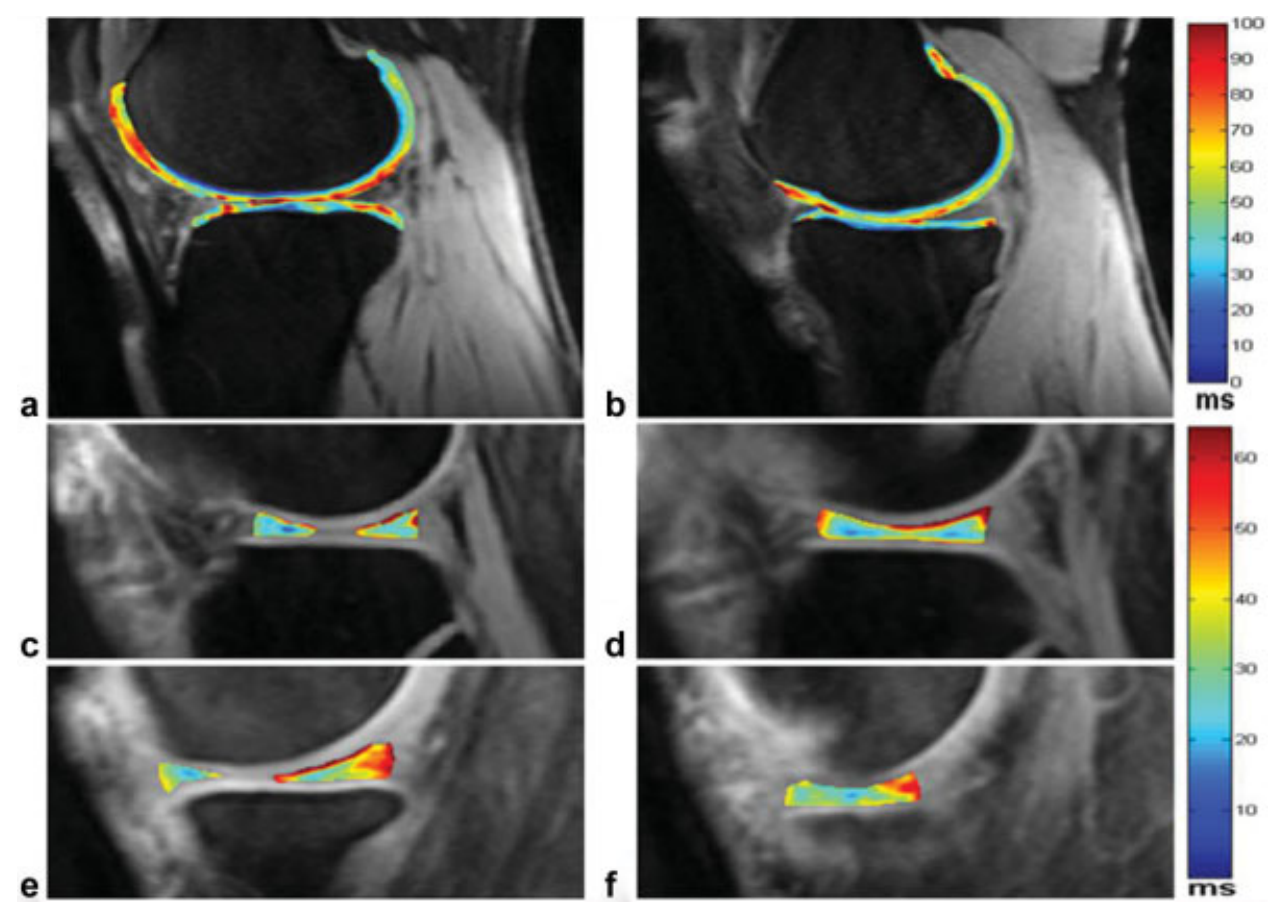

Fig. 5 Representative T1rho maps of cartilage in the (a) lateral and (b) medial compartments. T1rho maps of menisci in the (c, d) lateral and (e, f) medial compartments, respectively, obtained from a doubtful/minimal osteoarthritis patient. The color bars on the right show the T1 rho values ranges, respectively. The different bar scale ranges can display the respective T1rho values distribution of cartilage and meniscus more effectively. Reproduced with permission from Wang et al. ${ }^{95}$

to discriminate cartilage in OA patients from healthy cartilage, with FA having higher specificity. ${ }^{92}$ DTI was found to have high accuracy for detecting cartilage damage as well as for grading cartilage damage. ${ }^{93}$

\section{GAG Chemical Exchange Saturation Transfer Imaging}

Water exists in two states within cartilage, either bound to macromolecules or in the free water state. Water protons bound to macromolecules have unique RF frequency that can be saturated using off-resonance RF pulses. The bound water pool then interacts with the free water pool resulting in partial saturation of the free water pool. This effect can be measured to estimate local macromolecule content.
With gagCEST, off-resonance RF saturation pulses are designed specifically to saturate exchangeable protons residing on the hydroxyl groups of cartilage GAGs (-Fig. 5). This technique correlates well with ${ }^{23} \mathrm{Na}^{+}$imaging, and like ${ }^{23} \mathrm{Na}^{+}$ imaging, it is optimally performed at ultrahigh field strength (7 T) magnets. ${ }^{94}$

\section{Conclusion}

OA is the most prevalent joint disease in the United States with a tremendous socioeconomic burden. With efforts to develop a DMOAD for OA proving unsuccessful to date, it is clearly evident that imaging beyond radiography is needed for both clinical diagnoses of OA and for use as an outcome measure in OA research. MRI provides an unparalleled 
assessment of articular cartilage and has aptly been incorporated into the major clinical studies of OA including the OAI and the MOST. For the purpose of quantifying data from such trials, MRI-based semiquantitative grading systems for OA have been developed. The most widely used of these classification schemes include the WORMS and the BLOKS, with the MOAKS representing a hybrid of both these systems. In addition to the morphological evaluation, advanced MRI techniques have been developed to assess the biochemical composition of cartilage. These include relaxometry measurements (T2, T2*, and T1 $\rho$ mapping), sodium imaging, dGEMRIC, gagCEST, and DTI. These techniques have the potential to serve both as imaging biomarkers for OA and as quantitative, reproducible, and objective end points for $\mathrm{OA}$ research.

\section{Conflict of Interest \\ None declared.}

\section{References}

1 Centers for Disease Control and Prevention (CDC). Prevalence of doctor-diagnosed arthritis and arthritis-attributable activity limitation-United States, 2007-2009. MMWR Morb Mortal Wkly Rep 2010;59(39):1261-1265

2 Lawrence RC, Felson DT, Helmick CG, et al; National Arthritis Data Workgroup. Estimates of the prevalence of arthritis and other rheumatic conditions in the United States. Part II. Arthritis Rheum 2008;58(01):26-35

3 Altman R, Alarcón G, Appelrouth D, et al. The American College of Rheumatology criteria for the classification and reporting of osteoarthritis of the hip. Arthritis Rheum 1991;34(05):505-514

4 Boegård TL, Rudling O, Petersson IF, Jonsson K. Joint space width of the tibiofemoral and of the patellofemoral joint in chronic knee pain with or without radiographic osteoarthritis: a 2-year followup. Osteoarthritis Cartilage 2003;11(05):370-376

5 Guermazi A, Roemer FW, Felson DT, Brandt KD. Motion for debate: osteoarthritis clinical trials have not identified efficacious therapies because traditional imaging outcome measures are inadequate. Arthritis Rheum 2013;65(11):2748-2758

6 Bijlsma JW, Berenbaum F, Lafeber FP. Osteoarthritis: an update with relevance for clinical practice. Lancet 2011;377(9783):2115-2126

7 Tonge DP, Pearson MJ, Jones SW. The hallmarks of osteoarthritis and the potential to develop personalised disease-modifying pharmacological therapeutics. Osteoarthritis Cartilage 2014;22(05):609-621

8 Lane NE, Brandt K, Hawker G, et al. OARSI-FDA initiative: defining the disease state of osteoarthritis. Osteoarthritis Cartilage 2011; 19(05):478-482

9 Binks DA, Hodgson RJ, Ries ME, et al. Quantitative parametric MRI of articular cartilage: a review of progress and open challenges. $\mathrm{Br}$ J Radiol 2013;86(1023):20120163

10 Sophia Fox AJ, Bedi A, Rodeo SA. The basic science of articular cartilage: structure, composition, and function. Sports Health 2009;1(06):461-468

11 Buckwalter JA, Mankin HJ. Articular cartilage: degeneration and osteoarthritis, repair, regeneration, and transplantation. Instr Course Lect 1998;47:487-504

12 Rubenstein JD, Li JG, Majumdar S, Henkelman RM. Image resolution and signal-to-noise ratio requirements for MR imaging of degenerative cartilage. AJR Am J Roentgenol 1997;169(04):1089-1096

13 Cheng $\mathrm{Q}$ Z Zhao F-C. Comparison of 1.5- and 3.0-T magnetic resonance imaging for evaluating lesions of the knee: A systematic review and meta-analysis (PRISMA-compliant article). Medicine (Baltimore) 2018;97(38):e12401-e12401
14 Springer E, Bohndorf K, Juras V, et al. Comparison of routine knee magnetic resonance imaging at $3 \mathrm{~T}$ and $7 \mathrm{~T}$. Invest Radiol 2017;52 (01):42-54

15 Trattnig S, Ba-Ssalamah A, Pinker K, Plank C, Vecsei V, Marlovits S. Matrix-based autologous chondrocyte implantation for cartilage repair: noninvasive monitoring by high-resolution magnetic resonance imaging. Magn Reson Imaging 2005;23(07):779-787

16 Mugler JP III. Optimized three-dimensional fast-spin-echo MRI. J Magn Reson Imaging 2014;39(04):745-767

17 Kijowski R, Davis KW, Woods MA, et al. Knee joint: comprehensive assessment with $3 \mathrm{D}$ isotropic resolution fast spin-echo MR imaging-diagnostic performance compared with that of conventional MR imaging at 3.0 T. Radiology 2009;252(02):486-495

18 Jin J, Weber E, Destruel A, et al. An open 8-channel parallel transmission coil for static and dynamic 7T MRI of the knee and ankle joints at multiple postures. Magn Reson Med 2018;79 (03):1804-1816

19 Mohr A. The value of water-excitation 3D FLASH and fat-saturated PDw TSE MR imaging for detecting and grading articular cartilage lesions of the knee. Skeletal Radiol 2003;32(07):396-402

20 Blankenbaker DG, Ullrick SR, Kijowski R, et al. MR arthrography of the hip: comparison of IDEAL-SPGR volume sequence to standard MR sequences in the detection and grading of cartilage lesions. Radiology 2011;261(03):863-871

21 Regatte RR, Schweitzer ME. Novel contrast mechanisms at 3 Tesla and 7 Tesla. Semin Musculoskelet Radiol 2008;12(03):266-280

22 Outerbridge RE. The etiology of chondromalacia patellae. J Bone Joint Surg Br 1961;43-B:752-757

23 Noyes FR, Stabler CL. A system for grading articular cartilage lesions at arthroscopy. Am J Sports Med 1989;17(04):505-513

24 Brittberg M, Winalski CS. Evaluation of cartilage injuries and repair. J Bone Joint Surg Am 2003;85-A(Suppl 2):58-69

25 Palmieri-Smith RM, Thomas AC, Karvonen-Gutierrez C, Sowers MF. Isometric quadriceps strength in women with mild, moderate, and severe knee osteoarthritis. Am J Phys Med Rehabil 2010; 89(07):541-548

26 Hofmann GO, Marticke J, Grossstück R, et al. Detection and evaluation of initial cartilage pathology in man: a comparison between MRT, arthroscopy and near-infrared spectroscopy (NIR) in their relation to initial knee pain. Pathophysiology 2010;17 (01):1-8

27 Potter HG, Linklater JM, Allen AA, Hannafin JA, Haas SB. Magnetic resonance imaging of articular cartilage in the knee. An evaluation with use of fast-spin-echo imaging. J Bone Joint Surg Am 1998;80(09):1276-1284

28 Hunter DJ, Zhang YQ, Niu JB, et al. The association of meniscal pathologic changes with cartilage loss in symptomatic knee osteoarthritis. Arthritis Rheum 2006;54(03):795-801

29 Stehling C, Lane NE, Nevitt MC, Lynch J, McCulloch CE, Link TM. Subjects with higher physical activity levels have more severe focal knee lesions diagnosed with 3T MRI: analysis of a nonsymptomatic cohort of the osteoarthritis initiative. Osteoarthritis Cartilage 2010;18(06):776-786

30 Hovis KK, Alizai H, Tham SC, et al. Non-traumatic anterior cruciate ligament abnormalities and their relationship to osteoarthritis using morphological grading and cartilage T2 relaxation times: data from the Osteoarthritis Initiative (OAI). Skeletal Radiol 2012; 41(11):1435-1443

31 Hovis KK, Stehling C, Souza RB, et al. Physical activity is associated with magnetic resonance imaging-based knee cartilage T2 measurements in asymptomatic subjects with and those without osteoarthritis risk factors. Arthritis Rheum 2011;63 (08):2248-2256

32 Laberge MA, Baum T, Virayavanich W, et al. Obesity increases the prevalence and severity of focal knee abnormalities diagnosed using 3T MRI in middle-aged subjects-data from the Osteoarthritis Initiative. Skeletal Radiol 2012;41(06):633-641 
33 Virayavanich W, Alizai H, Baum T, et al. Association of frequent knee bending activity with focal knee lesions detected with 3T magnetic resonance imaging: data from the Osteoarthritis Initiative. Arthritis Care Res (Hoboken) 2013;65(09):1441-1448

34 Peterfy CG, Guermazi A, Zaim S, et al. Whole-Organ Magnetic Resonance Imaging Score (WORMS) of the knee in osteoarthritis. Osteoarthritis and cartilage/OARS. Osteoarthritis Cartilage 2004; 12(03):177-190

35 Hunter DJ, Lo GH, Gale D, Grainger AJ, Guermazi A, Conaghan PG. The reliability of a new scoring system for knee osteoarthritis MRI and the validity of bone marrow lesion assessment: BLOKS (Boston Leeds Osteoarthritis Knee Score). Ann Rheum Dis 2008; 67(02):206-211

36 Lynch JA, Roemer FW, Nevitt MC, et al. Comparison of BLOKS and WORMS scoring systems part I. Cross sectional comparison of methods to assess cartilage morphology, meniscal damage and bone marrow lesions on knee MRI: data from the osteoarthritis initiative. Osteoarthritis Cartilage 2010;18(11):1393-1401

37 Felson DT, Lynch J, Guermazi A, et al. Comparison of BLOKS and WORMS scoring systems part II. Longitudinal assessment of knee MRIs for osteoarthritis and suggested approach based on their performance: data from the Osteoarthritis Initiative. Osteoarthritis Cartilage 2010;18(11):1402-1407

38 Hunter DJ, Guermazi A, Lo GH, et al. Evolution of semi-quantitative whole joint assessment of knee OA: MOAKS (MRI Osteoarthritis Knee Score). Osteoarthritis Cartilage 2011;19(08):990-1002

39 Kornaat PR, Ceulemans RY, Kroon HM, et al. MRI assessment of knee osteoarthritis: Knee Osteoarthritis Scoring System (KOSS)inter-observer and intra-observer reproducibility of a compartment-based scoring system. Skeletal Radiol 2005;34(02):95-102

40 Rogers LQ, Macera CA, Hootman JM, Ainsworth BE, Blairi SN. The association between joint stress from physical activity and selfreported osteoarthritis: an analysis of the Cooper Clinic data. Osteoarthritis Cartilage 2002;10(08):617-622

41 Racunica TL, Teichtahl AJ, Wang Y, et al. Effect of physical activity on articular knee joint structures in community-based adults. Arthritis Rheum 2007;57(07):1261-1268

42 Stehling C, Liebl H, Krug R, et al. Patellar cartilage: T2 values and morphologic abnormalities at 3.0-T MR imaging in relation to physical activity in asymptomatic subjects from the Osteoarthritis Initiative. Radiology 2010;254(02):509-520

43 Stahl R, Luke A, Li X, et al. T1rho, T2 and focal knee cartilage abnormalities in physically active and sedentary healthy subjects versus early OA patients-a 3.0-Tesla MRI study. Eur Radiol 2009; 19(01):132-143

44 Washburn RA, Smith KW, Jette AM, Janney CA. The Physical Activity Scale for the Elderly (PASE): development and evaluation. J Clin Epidemiol 1993;46(02):153-162

45 Doré DA, Winzenberg TM, Ding C, et al. The association between objectively measured physical activity and knee structural change using MRI. Ann Rheum Dis 2013;72(07):1170-1175

46 Anandacoomarasamy A, Smith G, Leibman S, et al. Cartilage defects are associated with physical disability in obese adults. Rheumatology (Oxford) 2009;48(10):1290-1293

47 Baum T, Joseph GB, Nardo L, et al. Correlation of magnetic resonance imaging-based knee cartilage $\mathrm{T} 2$ measurements and focal knee lesions with body mass index: thirty-six-month followup data from a longitudinal, observational multicenter study. Arthritis Care Res (Hoboken) 2013;65(01):23-33

48 Roemer FW, Zhang Y, Niu J, et al; Multicenter Osteoarthritis Study Investigators. Tibiofemoral joint osteoarthritis: risk factors for MRdepicted fast cartilage loss over a 30-month period in the multicenter osteoarthritis study. Radiology 2009;252(03):772-780

49 Gudbergsen H, Boesen M, Lohmander LS, et al. Weight loss is effective for symptomatic relief in obese subjects with knee osteoarthritis independently of joint damage severity assessed by high-field MRI and radiography. Osteoarthritis Cartilage 2012;20(06):495-502
50 Ding C, Martel-Pelletier J, Pelletier JP, et al. Meniscal tear as an osteoarthritis risk factor in a largely non-osteoarthritic cohort: a cross-sectional study. J Rheumatol 2007;34(04):776-784

51 Lee DH, Lee BS, Kim JM, et al. Predictors of degenerative medial meniscus extrusion: radial component and knee osteoarthritis. Knee Surg Sports Traumatol Arthrosc 2011;19(02):222-229

52 Lee YG, Shim JC, Choi YS, Kim JG, Lee GJ, Kim HK. Magnetic resonance imaging findings of surgically proven medial meniscus root tear: tear configuration and associated knee abnormalities. J Comput Assist Tomogr 2008;32(03):452-457

53 Crema MD, Roemer FW, Felson DT, et al. Factors associated with meniscal extrusion in knees with or at risk for osteoarthritis: the Multicenter Osteoarthritis study. Radiology 2012;264(02): 494-503

54 Bloecker K, Guermazi A, Wirth W, et al; OAI investigators. Tibial coverage, meniscus position, size and damage in knees discordant for joint space narrowing-data from the Osteoarthritis Initiative. Osteoarthritis Cartilage 2013;21(03):419-427

55 Tanamas S, Hanna FS, Cicuttini FM, Wluka AE, Berry P, Urquhart DM. Does knee malalignment increase the risk of development and progression of knee osteoarthritis? A systematic review. Arthritis Rheum 2009;61(04):459-467

56 Felson DT, Niu J, Gross KD, et al. Valgus malalignment is a risk factor for lateral knee osteoarthritis incidence and progression: findings from the Multicenter Osteoarthritis Study and the Osteoarthritis Initiative. Arthritis Rheum 2013;65(02):355-362

57 Sharma L, Chmiel JS, Almagor O, et al. The role of varus and valgus alignment in the initial development of knee cartilage damage by MRI: the MOST study. Ann Rheum Dis 2013;72(02):235-240

58 Krug R, Stehling C, Kelley DA, Majumdar S, Link TM. Imaging of the musculoskeletal system in vivo using ultra-high field magnetic resonance at 7 T. Invest Radiol 2009;44(09):613-618

59 Jordan CD, Saranathan M, Bangerter NK, Hargreaves BA, Gold GE. Musculoskeletal MRI at 3.0 T and 7.0 T: a comparison of relaxation times and image contrast. Eur J Radiol 2013;82(05):734-739

60 Pruessmann KP. Parallel imaging at high field strength: synergies and joint potential. Top Magn Reson Imaging 2004;15(04):237-244

61 Regatte RR, Schweitzer ME. Ultra-high-field MRI of the musculoskeletal system at 7.0T.J Magn Reson Imaging 2007;25(02):262-269

62 Peterfy CG, Schneider E, Nevitt M. The Osteoarthritis Initiative: report on the design rationale for the magnetic resonance imaging protocol for the knee. Osteoarthritis Cartilage 2008;16(12): 1433-1441

63 Liess C, Lüsse S, Karger N, Heller M, Glüer CC. Detection of changes in cartilage water content using MRI T2-mapping in vivo. Osteoarthritis Cartilage 2002;10(12):907-913

64 Carballido-Gamio J, Blumenkrantz G, Lynch JA, Link TM, Majumdar S. Longitudinal analysis of MRI T(2) knee cartilage laminar organization in a subset of patients from the Osteoarthritis Initiative. Magn Reson Med 2010;63(02):465-472

65 Joseph GB, Baum T, Alizai H, et al. Baseline mean and heterogeneity of MR cartilage T2 are associated with morphologic degeneration of cartilage, meniscus, and bone marrow over 3 years-data from the Osteoarthritis Initiative. Osteoarthritis Cartilage 2012;20(07): 727-735

66 Chang G, Xia D, Sherman O, et al. High resolution morphologic imaging and T2 mapping of cartilage at 7 Tesla: comparison of cartilage repair patients and healthy controls. MAGMA 2013;26 (06):539-548

67 Domayer SE, Apprich S, Stelzeneder D, et al. Cartilage repair of the ankle: first results of T2 mapping at 7.0 T after microfracture and matrix associated autologous cartilage transplantation. Osteoarthritis Cartilage 2012;20(08):829-836

68 Welsch GH, Mamisch TC, Hughes T, et al. In vivo biochemical 7.0 Tesla magnetic resonance: preliminary results of dGEMRIC, zonal $\mathrm{T} 2$, and $\mathrm{T}^{*}$ mapping of articular cartilage. Invest Radiol 2008;43(09):619-626 
69 Welsch GH, Mamisch TC, Marlovits S, et al. Quantitative T2 mapping during follow-up after matrix-associated autologous chondrocyte transplantation (MACT): full-thickness and zonal evaluation to visualize the maturation of cartilage repair tissue. J Orthop Res 2009;27(07):957-963

70 Mamisch TC, Hughes T, Mosher TJ, et al. T2 star relaxation times for assessment of articular cartilage at $3 \mathrm{~T}$ : a feasibility study. Skeletal Radiol 2012;41(03):287-292

71 Welsch GH, Apprich S, Zbyn S, et al. Biochemical (T2, T2* and magnetisation transfer ratio) MRI of knee cartilage: feasibility at ultra-high field (7T) compared with high field (3T) strength. Eur Radiol 2011;21(06):1136-1143

72 Singh A, Haris M, Cai K, Kogan F, Hariharan H, Reddy R. High resolution T1 $\rho$ mapping of in vivo human knee cartilage at 7T. PLoS One 2014;9(05):e97486

73 Sepponen RE, Pohjonen JA, Sipponen JT, Tanttu JI. A method for T1 rho imaging. J Comput Assist Tomogr 1985;9(06):1007-1011

74 Keenan KE, Besier TF, Pauly JM, et al. Prediction of glycosaminoglycan content in human cartilage by age, T1 $\rho$ and T2 MRI. Osteoarthritis Cartilage 2011;19(02):171-179

75 Wyatt C, Guha A, Venkatachari A, et al. Improved differentiation between knees with cartilage lesions and controls using 7T relaxation time mapping. J Orthop Translat 2015;3(04):197-204

76 Prasad AP, Nardo L, Schooler J, et al. T(1)rho and T(2) relaxation times predict progression of knee osteoarthritis. Osteoarthritis and cartilage/OARS. Osteoarthritis Research Society 2013;21:69-76

77 Bae WC, Du J, Bydder GM, Chung CB. Conventional and ultrashort time-to-echo magnetic resonance imaging of articular cartilage, meniscus, and intervertebral disk. Top Magn Reson Imaging 2010; 21(05):275-289

78 Bae WC, Dwek JR, Znamirowski R, et al. Ultrashort echo time MR imaging of osteochondral junction of the knee at $3 \mathrm{~T}$ : identification of anatomic structures contributing to signal intensity. Radiology 2010;254(03):837-845

79 Chang EY, Pallante-Kichura AL, Bae WC, et al. Development of a Comprehensive Osteochondral Allograft MRI Scoring System (OCAMRISS) with histopathologic, micro-computed tomography, and biomechanical validation. Cartilage 2014;5(01):16-27

80 Chu CR, Williams AA, West RV, et al. Quantitative magnetic resonance imaging UTE-T2* mapping of cartilage and meniscus healing after anatomic anterior cruciate ligament reconstruction. Am J Sports Med 2014;42(08):1847-1856

81 Lazik A, Korsmeier K, Classen T, et al. 3 Tesla high-resolution and delayed gadolinium enhanced MR imaging of cartilage (dGEMRIC) after autologous chondrocyte transplantation in the hip. J Magn Reson Imaging 2015;42(03):624-633
82 Watanabe A, Wada Y, Obata T, et al. Delayed gadolinium-enhanced MR to determine glycosaminoglycan concentration in reparative cartilage after autologous chondrocyte implantation: preliminary results. Radiology 2006;239(01):201-208

83 d'Entremont AG, McCormack RG, Agbanlog K, et al. Cartilage health in high tibial osteotomy using dGEMRIC: relationships with joint kinematics. Knee 2015;22(03):156-162

84 Schleich C, Müller-Lutz A, Sewerin P, et al. Intra-individual assessment of inflammatory severity and cartilage composition of finger joints in rheumatoid arthritis. Skeletal Radiol 2015;44 (04):513-518

85 Owman H, Tiderius CJ, Ericsson YB, Dahlberg LE. Long-term effect of removal of knee joint loading on cartilage quality evaluated by delayed gadolinium-enhanced magnetic resonance imaging of cartilage. Osteoarthritis Cartilage 2014;22(07):928-932

86 Madelin G, Lee JS, Regatte RR, Jerschow A. Sodium MRI: methods and applications. Prog Nucl Magn Reson Spectrosc 2014;79:14-47

87 Trattnig S, Welsch GH, Juras V, et al. 23Na MR imaging at $7 \mathrm{~T}$ after knee matrix-associated autologous chondrocyte transplantation preliminary results. Radiology 2010;257(01):175-184

88 Madelin G, Babb J, Xia D, et al. Articular cartilage: evaluation with fluid-suppressed 7.0-T sodium MR imaging in subjects with and subjects without osteoarthritis. Radiology 2013;268(02): 481-491

89 Wiggins GC, Brown R, Lakshmanan K. High-performance radiofrequency coils for (23)Na MRI: brain and musculoskeletal applications. NMR Biomed 2016;29(02):96-106

90 Zbýň Š, Brix MO, Juras V, et al. Sodium magnetic resonance imaging of ankle joint in cadaver specimens, volunteers, and patients after different cartilage repair techniques at $7 \mathrm{~T}$ : initial results. Invest Radiol 2015;50(04):246-254

91 Chang G, Madelin G, Sherman OH, et al. Improved assessment of cartilage repair tissue using fluid-suppressed ${ }^{23} \mathrm{Na}$ inversion recovery MRI at 7 Tesla: preliminary results. Eur Radiol 2012; 22(06):1341-1349

92 Raya JG, Horng A, Dietrich O, et al. Articular cartilage: in vivo diffusion-tensor imaging. Radiology 2012;262(02):550-559

93 Raya JG, Melkus G, Adam-Neumair S, et al. Diffusion-tensor imaging of human articular cartilage specimens with early signs of cartilage damage. Radiology 2013;266(03):831-841

94 Singh A, Haris M, Cai K, et al. Chemical exchange saturation transfer magnetic resonance imaging of human knee cartilage at $3 \mathrm{~T}$ and $7 \mathrm{~T}$. Magn Reson Med 2012;68(02):588-594

95 Wang L, Chang G, Xu J, et al. T1 rho MRI of menisci and cartilage in patients with osteoarthritis at 3T. Eur J Radiol 2012;81(09): 2329-2336 